\title{
Layer resolved magnetic domain imaging of epitaxial heterostructures in large applied magnetic fields
}

\author{
S. Zohar ${ }^{1}$, Y. Choi ${ }^{1}$, D.M. Love², R. Mansell², C.H.W. Barnes ${ }^{2}$, D.J. Keavney ${ }^{1}$, R.A. Rosenberg ${ }^{1}$ \\ ${ }^{1}$ Advanced Photon Source, Argonne National Laboratory, Argonne, Illinois 60439, United States \\ ${ }^{2}$ Cavendish Laboratory, University of Cambridge, J J Thomson Avenue, CB3 OHE Cambridge, United Kingdom
}

We use X-ray Excited Luminescence Microscopy to investigate the elemental and layer resolved magnetic reversal in an interlayer exchange coupled (IEC) epitaxial $\mathrm{Fe} / \mathrm{Cr}$ wedge/Co heterostructure. The transition from strongly coupled parallel Co-Fe reversal for Cr thickness $\mathrm{t}_{\mathrm{Cr}}<0.34 \mathrm{~nm}$ to weakly coupled layer independent reversal for $\mathrm{t}_{\mathrm{Cr}}>1.5 \mathrm{~nm}$ is punctuated at $0.34<\mathrm{t}_{\mathrm{Cr}}<1.5 \mathrm{~nm}$ by a combination of IEC guided domain wall motion and stationary zig zag domain walls. Domain walls nucleated at switching field minima are guided by IEC spatial gradients and collapse at switching field maxima.

\section{INTRODUCTION}

Magnetic heterostructures are of great interest owing to their applications in magnetic memory, hybrid semiconductor-ferromagnetic technology, and quantum information. The physics enabling such versatile functionality stems from spin dependent reflectivity ${ }^{1}$ at the ferromagnetic-normal metal interface that gives rise to interlayer exchange coupling (IEC), magneto resistance, and spin transfer torque. From among these effects, IEC is a particularly intriguing phenomenon described by preferred parallel/anti-parallel alignment of two ferromagnetic (FM) layers that oscillates with the thickness of a separating spacer layer ${ }^{2,3}$. In recent years, magnetic multilayers have been investigated using magnetic contrast $\mathrm{x}$-ray imaging techniques. These studies showed that stray fields from domain walls can modify the neighboring layer domain structure ${ }^{4}$, identified contributions from intrinsic and coupled effects ${ }^{5}$, and imaged injection locking of vortex core gyration with 30 nm resolution ${ }^{6}$. These studies, however, were restricted to a combination of polycrystalline samples, remanence magnetization, and small pulsed fields due to the required x-ray transparent membranes for transmission x-ray microscopy (TXM) and small fields required for photoemission electron microscopy (PEEM). To date, there have 
been no layer resolved magnetic imaging studies of epitaxial structures in magnetic fields of several hundred Gauss. Such capabilities will be important for future studies investigating integrated multilayered ${ }^{7}$ hybrid ferromagnetic semiconductor devices ${ }^{8}$ and will enable isolation of the fundamental physics from domain and defect driven effects ${ }^{1}$.

In this letter, we demonstrate layer resolved domain imaging of an epitaxial $\mathrm{Fe}(10 \mathrm{~nm}) / \mathrm{Cr}(0-4 \mathrm{~nm}) / \operatorname{Co}(10$ $\mathrm{nm}$ ) wedge structure deposited on GaAs in applied magnetic field > $400 \mathrm{G}$ using X-ray Excited Luminescent Microscopy (XELM) ${ }^{9}$. This system is of interest due to demonstrated electrical spin injection from the Fe layer into GaAs and the presence of a coupled electronic-nuclear spin system found in $\mathrm{GaAs}^{8,10}$. In recent years, studies of $\mathrm{Fe} / \mathrm{Cr} / \mathrm{Co}$ structures deposited on $\mathrm{MgO}$ showed that the Co magnetization has fourfold-anisotropy ${ }^{11}$ and provided evidence for both short and long period Cr thickness dependent oscillations. Further analysis in Ref. 11 was complicated by suspected formation of domains. Here, we use XELM to demonstrate the presence of unique $\mathrm{Cr}$ thickness dependent domain structure in both $\mathrm{Co}$ and $\mathrm{Fe}$ as proposed in Ref. ${ }^{11}$. For strong ferromagnetic IEC at $\mathrm{Cr}$ thickness $\mathrm{t}_{\mathrm{Cr}}<0.34 \mathrm{~nm}$, the $\mathrm{Fe}$ and $\mathrm{Co}$ magnetizations are parallel for all applied magnetic fields with switching described by domain wall motion. For $\mathrm{t}_{\mathrm{Cr}}>1.5 \mathrm{~nm}$, the IEC is much weaker and is well approximated by an effective applied magnetic field. The Fe reversal is adequately described by the macro moment approximation while the Co nucleation field is shifted by an IEC induced effective applied magnetic field. For Cr thickness in the range 0.5 -1.5 nm, we observe spatial IEC gradients that guide domain walls nucleated at coercivity minima toward coercivity maxima where the domains collapse. The Fe and Co switching fields oscillate with $\sim 2 \mathrm{~nm} \mathrm{t}_{\mathrm{Cr}}$ period.

XELM is a powerful imaging method that uses the element sensitivity of $\mathrm{x}$-ray circular dichroism to image the magnetization. In this technique, the underlying substrate serves as both a scintillator whose luminescence is used to form an image of the x-ray transmission as well as a lattice matched support enabling epitaxial deposition of the magnetic layers. In heterostructures where each layer contains a unique element, the elemental resolving power maps to layer resolution. XELM is well suited for studying epitaxial heterostructures, as its ability to image with elemental and magnetic contrast is not limited to samples with $\mathrm{x}$-ray transparent substrates necessary for TXM ${ }^{6}$ or weak pulsed fields required for PEEM ${ }^{5,4}$. 


\section{Experiment}

An $\mathrm{Fe}(10 \mathrm{~nm}) / \mathrm{Cr}(0-4 \mathrm{~nm}) / \mathrm{Co}(10 \mathrm{~nm})$ epitaxial tri-layer was grown on n:GaAs (100) using MBE deposition with a base pressure of $3 \times 10^{-10}$ torr at a deposition rate of $0.4 \mathrm{~nm} / \mathrm{min}$ and at $150^{\circ} \mathrm{C}$. A $7 \mathrm{~mm}$ long $\mathrm{Cr}$ wedge was made using a linear shutter and $3 \mathrm{~nm} \mathrm{MgO}$ cap was deposited at $110^{\circ} \mathrm{C}$. The GaAs substrate was annealed prior to deposition at $600^{\circ} \mathrm{C}$ in a separate chamber with a base pressure of $4 \times 10^{-10}$ torr for one hour for oxide removal and surface annealing. Epitaxial deposition was verified by RHEED and depositions rates were measured using a crystal monitor. Fe growth on GaAs (100) is in the BCC structure ${ }^{12}$ with crystalline (11̄0) and (110) in the plane of the film as shown in Figure 1(b). Cr growth on Fe is in the BCC (100) structure as well ${ }^{11}$ and the wedge gradient is parallel to the (11̄0) direction. The first $3 \mathrm{~nm}$ of Co grows in the HCP structure with the $\mathrm{C}$ (hard) axis randomly oriented parallel with the Fe (110) and (110) planes ${ }^{11,13}$. Similar Co growth on $\mathrm{Cr}$ has been found for Co thickness up to $100 \mathrm{~nm}^{14}$. XMCD reflectivity and XELM measurements were performed at beamline 4-ID-C of the Advanced Photon Source on the Fe and $\mathrm{Co}_{3}$ edges with an applied external magnetic field directed parallel to the Fe (110) direction. The $1.5 \mathrm{eV}(850 \mathrm{~nm})$ luminescence from the GaAs substrate, cooled to $100 \mathrm{~K}$, was imaged onto a CCD element using a Questar QM 100 (Company 7) long range microscope. The experimental setup and sample structure are schematically shown in Figure 1. Sequences of images were taken at magnetic fields ranging from 0 to $500 \mathrm{G}$ in increments of $15 \mathrm{G}$ and arranged into videos (Figure 2(a-f) multimedia view). Representative frames from the videos, shown in Figures 2(a-f), were selected to highlight: 1) IEC guided domain wall motion, 2) the Co transition to nucleation, and 3) Fe zig zag domain walls. Magnetization curves extracted from the images by graphing the pixel intensity against the applied field are shown in Figure 3(a).

\section{Results}

In Figure 2(h), we show the elemental specific XMCD magnetization curves measured in reflectivity. For magnetic fields applied along the Fe (110) axis, all switching fields vary with Cr thickness. The nature of reversal (coherent rotation vs domain) cannot be determined within the resolution of the $\mathrm{x}$-ray beam size $(300 \mu \mathrm{m} \mathrm{x}$ 
$100 \mu \mathrm{m}$ ). To determine the magnetic reversal process, we use XELM to image the magnetic structure (Figure 2 (af) multimedia view).

The observed magnetic domains exhibit a rich variety of behavior that can be divided into three categories: 1) strong IEC coupling for Cr thickness $<0.34 \mathrm{~nm}, 2)$ intermediate IEC coupling for Cr thickness 0.34 $\mathrm{nm}<\mathrm{t}_{\mathrm{Cr}}<1.5 \mathrm{~nm}$, and 3) weak coupling for Cr thickness $>1.5 \mathrm{~nm}$. In figures $2(\mathrm{a}-\mathrm{b})$ the magnetic domain structures for strongly ferromagnetic IEC with $\mathrm{t}_{\mathrm{Cr}}<0.34 \mathrm{~nm}$ are shown. The magnetic domains are zig zag shaped and protrude from the defects towards the right hand side of the image. In figures 2(c-d) the magnetic domain structures for intermediate IEC with $0.36<\mathrm{t}_{\mathrm{Cr}}<0.64 \mathrm{~nm}$ are shown. The magnetic domain walls here have a combination of smaller zig zag and smooth walls that propagate along the $\mathrm{Fe}(1 \overline{1} 0)$ direction. The dashed lines are the location from which the hysteresis loop's ascending branch shown in Figure 3(a) were taken. The hysteresis curve step size is $>15 \%$, consistent with previously reported XMCD values for Fe and Co ${ }^{15}$. In Figure 2(e-f) the magnetic domain structures for weak IEC with $\mathrm{t}_{\mathrm{Cr}}>1.5 \mathrm{~nm}$ are shown. Here, a non-propagating zig zag wall is observed in the Fe layer. The Co reversal mechanisms are different on either side of the Fe zig zag domain wall, changing from domain wall motion above the domain wall, to nucleation below the domain wall. Both the Fe and Co switching fields shown in Figure 3(b) oscillate with the Cr thickness as expected for interlayer exchanged coupled systems.

\section{Discussion}

To understand the effects of coupling on the magnetization and switching field, we recall the macromoment approximation for the magnetic free energy, $F_{\text {mag }}$, of an exchange coupled tri-layer thin film structure ${ }^{11}$ :

$$
\begin{aligned}
& F_{m a g}= \\
& \left(-\mu_{0} M{ }^{F e} H \cos \left(\Phi_{F e}-\Phi_{H}\right)+K_{1}^{F e} \sin ^{2} \Phi_{F e} \cos ^{2} \Phi_{F e}\right) t F^{+} \\
& \left(-\mu_{0} M^{C o} H \cos \left(\Phi_{C o}-\Phi_{H}\right)+K_{2}^{C o} \sin ^{2} \Phi_{C o} \cos ^{2} \Phi_{C o}\right) C_{C o}+ \\
& -2 A_{12} \cos \left(\Phi_{F e}-\Phi_{C o}\right)-2 B_{12} \cos ^{2}\left(\Phi_{F e}-\Phi_{C o}\right)
\end{aligned}
$$


where $M$ is the magnetization, $t$ is the layer thickness, $\Phi$ is the angle of the sample magnetization, $H$ is the applied magnetic field, $K$ is the crystalline anisotropy constant, and the super and sub scripts identify the layer. The $\mathrm{A}_{12}$ and $\mathrm{B}_{12}$ terms are the bilinear and bi-quadratic interlayer exchange coupling terms respectively. The bilinear interlayer exchange coupling has an oscillatory $\mathrm{Cr}$ thickness dependence, $\mathrm{A}_{12} \propto \sin \left(2 \pi \mathrm{t}_{\mathrm{Cr}} / \lambda+\phi\right)^{11,3,2,1}$, where $\lambda$ is the oscillation period. The $\mathrm{Cr}$ thickness is linearly dependent on $\hat{y}, t_{\mathrm{Cr}}=\alpha \cdot y$, where $\alpha$ is the wedge gradient. Combining these expressions yields $\mathrm{A}_{12} \propto \sin (2 \pi \alpha \cdot \mathrm{y} / \lambda+\phi)$. Inserting this expression for $\mathrm{A}_{12}$ into equation 1 results in $\mathrm{F}_{\mathrm{mag}}$ oscillating along $\hat{\mathrm{y}}$ with equal energy contours along $\hat{\mathrm{x}}$.

Equation 1 does not include terms for intra-layer exchange and magneto-static dipolar interactions. The intra-layer exchange energy originates from the exchange interaction between neighboring atoms and favors uniform magnetization and larger domains. The magneto-static energy originates from the magnetic dipole interaction between different regions within the sample ${ }^{16}$ and favors closed loop magnetization flux configurations. In the macro-moment approximation, the magnetization is uniform and the shape anisotropy arising from dipolar interactions is implicit by restriction of the magnetization parallel to the thin film plane. When the macro-moment approximation is not applicable, the magneto-static, intra-layer exchange, crystalline anisotropy, and IEC, compete with each other resulting in a wide variety of different domain structures ${ }^{17} 16$.

In the thin Cr spacer limit, $\mathrm{t}_{\mathrm{Cr}}<0.34 \mathrm{~nm}$ (Figure 2(a-b)), the Fe and Co domain structure are identical indicating strong ferromagnetic pinhole coupling that keeps both layers parallel for all applied fields (i.e. $\left.\Phi_{\mathrm{Fe}}=\Phi_{\mathrm{Co}}\right)$. Here, the system behaves as a single layer with a single effective anisotropy constant. We observe the magnetization reversal in this region is caused by domain wall motion which reduces the Co coercivity away from high field nucleation processes. The zig zag domains point parallel to the applied field reducing magnetic charge density from head on head magnetization ${ }^{16}$.

In the thick Cr layer limit, $\mathrm{t}_{\mathrm{Cr}}>1.5 \mathrm{~nm}$, the IEC coupling is sufficiently weak for the reversal mechanism to be accurately described by uncoupled Fe and Co reversal: the Fe switching is caused by a single moving smooth domain wall while the Co reverses by a higher density of nucleating domains ${ }^{18}$. Since the Co and $\mathrm{Fe}$ magnetizations in this Cr thickness range are nearly collinear for magnetic fields several tens of Gauss above and 
below their switching fields, the IEC in equation 1 can be approximated as an applied magnetic field, $\mathrm{H}_{\mathrm{eff}}$, that offsets the switching field by

$$
\mathrm{H}_{\mathrm{eff}}=2 \mathrm{~A}_{12} /\left(\mu_{0} M^{\mathrm{Fe}} t_{\mathrm{FM}}\right)(2)
$$

Given knowledge of $\mathrm{H}_{\text {eff }}$, this expression allows us to calculate $\mathrm{A}_{12}$. For $\mathrm{t}_{\mathrm{Cr}}>1.5 \mathrm{~nm}$, we can approximate $\mathrm{H}_{\text {eff }}$ as the difference between the thick $\mathrm{Cr}$ switching field and the thin $\mathrm{Cr}$ switching field, $\mathrm{H}_{\text {eff }}=\mathrm{H}_{\mathrm{S}, \mathrm{FM}}\left(\mathrm{t}_{\mathrm{Cr}}=4 \mathrm{~nm}\right)-\mathrm{H}_{\mathrm{S}, \mathrm{FM}}\left(\mathrm{t}_{\mathrm{Cr}}\right)$ for the respective FM layer, where $\mathrm{H}_{\mathrm{S}, \mathrm{FM}}\left(\mathrm{t}_{\mathrm{Cr}}\right)$ is the switching field. A separate evaluation of the exchange energy using equation 2 on both the Co and Fe layers is self-consistent for $1 \mathrm{~nm}<\mathrm{t}_{\mathrm{Cr}}<$ $3 \mathrm{~nm}$ within an offset step error. $\mathrm{A}_{12}$ peaks with a value of $0.1 \mathrm{ergs} / \mathrm{cm}^{2}$ at the local extreme of $2 \mathrm{~nm} \mathrm{t}_{\mathrm{Cr}}$.

For intermediate $\mathrm{Cr}$ thickness, $0.36 \mathrm{~nm}<\mathrm{t}_{\mathrm{Cr}}<1.5 \mathrm{~nm}$, IEC spatial gradients guide the domain walls along $\hat{y}$ (Figures 2(c-d) (multimedia view)). The domain walls lie approximately parallel to the equal energy contours along $\hat{x}$ that are predicted when equation 1 is applied to a tri-layer with a wedge shaped spacer. As the applied magnetic field is ramped, the Fe domain nucleates far to the left and propagates to right. The location of this initial switching corresponds to the Fe switching field minimum in Figure 3(b) (inset). The domain continues to expand by propagating their walls along the $\hat{y}$ direction with increasing magnetic field, while staying nearly parallel to the equal energy. We can understand this behavior as follows. From equations 1 and 2, the total effective magnetic field, $\mathrm{H}+\mathrm{H}_{\text {eff, }}$ oscillates with the $\mathrm{Cr}$ thickness. Since the $\mathrm{Cr}$ thickness depends on $\hat{\mathrm{y}}$, the switching field is shifted for different sections of the Fe and Co layers displaced along the wedge gradient (i.e. $\hat{y}$ ). Displacements along $\hat{x}$ see the same effective field and thus have the same switching field. In the bottom of Figure 2(c) (multimedia view) the Fe domain walls converge from both the top and the bottom until the domain completely collapses. The location of the collapse corresponds to the Fe switching field maxima shown in Figure 3(b). Similar domain collapse from converging domain walls can be observed in the Co layer at $\mathrm{t}_{\mathrm{Cr}}=1.3 \mathrm{~nm}{ }^{18}$.

Regions where IEC ceases to guide domain walls, such as in Figures 2(d) and 2(e), are particularly interesting. In Figure 2(d) we show the Co domain structure at the location of the corresponding dark region in the 
Fe layer (Figure 2(c)). We interpret the intermediate contrast level to indicate the formation of a $90^{\circ}$ domain. The Co domain becomes progressively more visible with increasing field as the magnetization flops away from the hard axis, driven by the coupling to the Fe layer as expected for coupled fourfold anisotropy systems ${ }^{19}$. The transition between the intermediate and weak coupling occurring between $1.52 \mathrm{~nm}-1.6 \mathrm{~nm} \mathrm{Cr}$ is separated by a zig zag domain (red enclosed section of Figure 2(e)). In this $t_{\mathrm{Cr}}$ range, there is an 80 Gauss jump in the Fe switching field's $t_{\mathrm{Cr}}$ dependence (Figure 3(b)). Additionally, this is same $\mathrm{t}_{\mathrm{Cr}}$ where the Co reversal mechanism changes from domain wall motion to nucleation (Figure 2(f)). Compared to the domain walls for $\mathrm{t}_{\mathrm{Cr}}<0.34 \mathrm{~nm}$, this zig zag domain wall geometry has a component that points along $\hat{y}$ as shown in Figure $2(\mathrm{~g})$. Here, the crystalline anisotropy rotates the magnetization towards an easy axis, creating a magnetic dipole disfavored head on head component. Since the IEC here at thicker $\mathrm{Cr}$ is insufficient to force the domain parallel to the equal IEC energy contour, the wall folds into a zig zag to reduce the head on head magneto-static charge density.

\section{Conclusion}

In conclusion we have imaged the layer resolved magnetization field dependence of an epitaxial IEC $\mathrm{Fe} / \mathrm{Cr} / \mathrm{Co}$ heterostructure using XELM. The switching field oscillates with the $\mathrm{Cr}$ thickness, as expected for interlayer exchange coupled heterostructures. We observe the $\mathrm{Cr}$ thickness dependent reversal mechanism transitions between nucleation, domain wall motion, and IEC guided domain wall propagation. This work demonstrates XELM's ability to image layer resolved magnetization field dependence in epitaxial systems and will enable the investigation of integrated spintronic devices possessing both ferromagnetic and semiconductor functionality.

RM acknowledges funding from the European Community under the Seventh Framework Program Contract No. 247368: 3SPIN. DL acknowledges funding from the EPSRC. The work performed at the Advanced Photon Source was supported by the U.S. Department of Energy, Office of Science, Office of Basic Energy Sciences under Contract No. DE-AC02-06CH11357. 


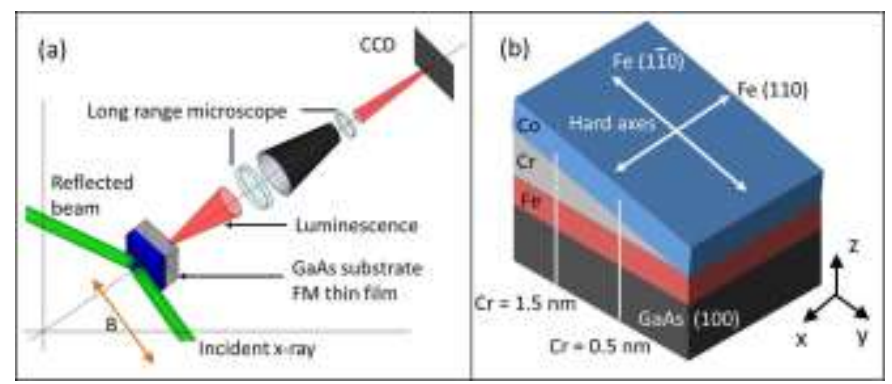

FIG. 1. (a) Incident circularly polarized $\mathrm{x}$-rays are absorbed by the magnetic over-layer. The transmission is detected by imaging luminescence from the GaAs substrate. A magnetic field is applied parallel to the incident beam and the luminescence is focused onto a CCD using a long range microscope. The sample plane normal vector forms a $77^{\circ}$ angle with respect to the $\mathrm{x}$-ray beam. The difference between two images taken at opposite $\mathrm{x}$ ray helicity provides the magnetic contrast. (b) Illustration of the asymmetric tri-layer. The applied magnetic field is parallel to the $\mathrm{Fe}(110)$ axis. The Fe (110) and (1) 10$)$ are parallel to the $\hat{\mathbf{x}}$ and $\hat{\mathbf{y}}$ axes respectively and are consistent with coordinate axes throughout the remaining figures. 


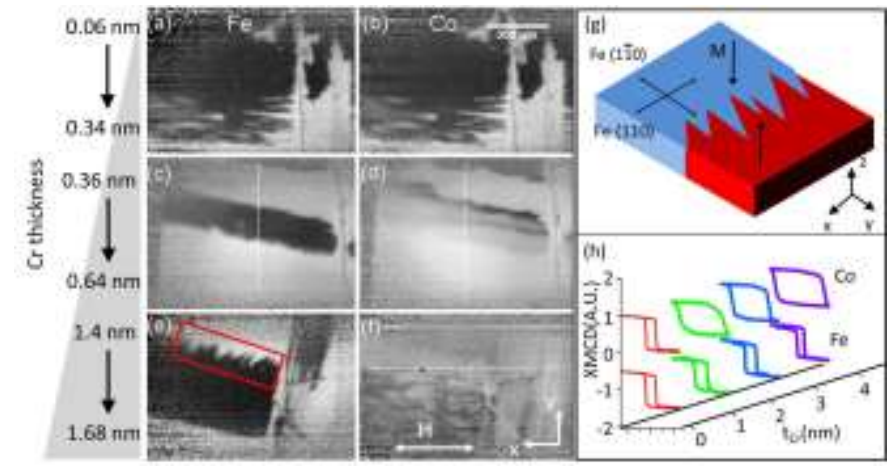

FIG. 2. In panels (a-f) we show magnetic contrast images of the Fe and Co layers at magnetic fields of: $105 \mathrm{G}$ for panels (a- b), $130 \mathrm{G}$ for panels (c-d), $90 \mathrm{G}$ for panel (e), and $360 \mathrm{G}$ for panel (f). The light and dark regions correspond to oppositely aligned magnetizations (multimedia view). The opaque grey triangle on the left hand side indicates the $\mathrm{Cr}$ thickness with the adjacent panel. In panels (a-b), strong pin-hole coupling gives rise to identical zig zag domain in both layers. In panels (c-d) the FM coupled and IEC spatial gradients drive domain wall motion along the y direction. The intermediate gray in (d) indicates a 90 degree domain in the Co layer that occurs in the same location as the dark shaded region of the Fe (panel (c). The dashed lines are the locations from which the magnetization curves in figure 3(a) are taken. In panels (e) we show the magnetic structure occurring at the 80 Gauss jump in the Fe switching field (Figure 3(b)). This Cr thickness, $\mathrm{t}_{\mathrm{Cr}}=1.5 \mathrm{~nm}$, coincides with the Co transition from domain wall motion to nucleation and is marked by a dashed line in panel (f). (g) Illustration of an ideal zig zag domain wall for the domain structure in panel (e). (h) Magnetization hysteresis curves acquired using $\mathrm{XMCD}$ in reflectivity sampled at several positions along the $\mathrm{Cr}$ wedge. 


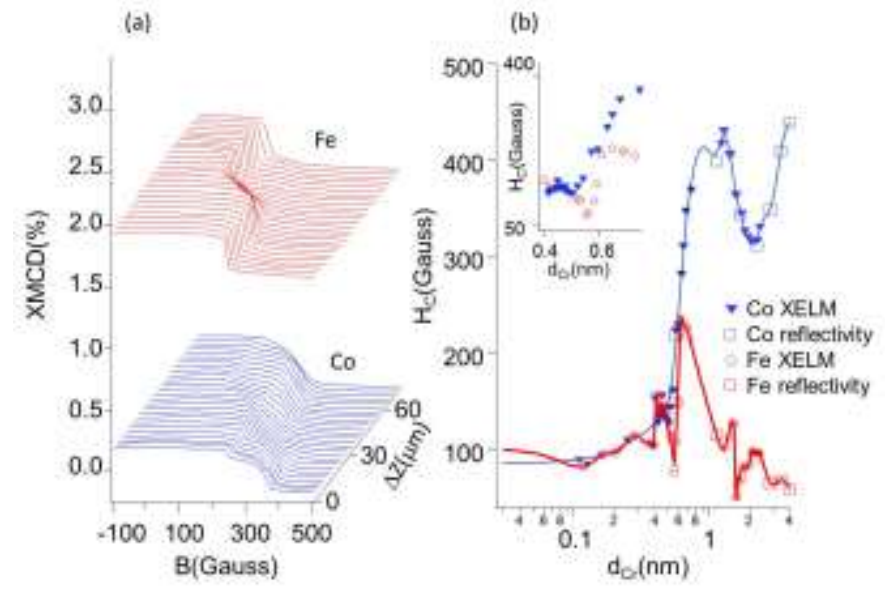

FIG. 3. (a) Representative hysteresis loop's ascending branches taken from points along the dashed lines near the domain wall in Figures 2(c-d). Small vertical offsets between magnetization curves were corrected using median image filtering. (b) Switching field $\mathrm{tCr}$ dependence extracted from images sequences. The Co maximum and minimum just above $1 \mathrm{~nm}$ and $2 \mathrm{~nm}$ respectively implies a periodicity of $\sim 2 \mathrm{~nm}$. 


\section{Bibliography}

1 B. Heinrich, J. A. Bland and M. D. Stiles, Ultrathin Magnetic Structures III, Berlin Heidelberg New York: Springer, 2005.

2 S. S. Parkin, N. More and K. P. Roche, Physical Review Letters, 64, 2304 (1989).

3 S. S. Parkin, R. Bhadra and K. P. Roche, Physical Review Letters, 66, 2152 (1991).

4 W. Kuch, L. I. Chelaru, K. Fukumoto, F. Porrati, F. Offi, M. Kostugi and J. Kirschner, Physical Review B, 67, 214403 (2003).

5 J. Vogel, W. Kuch, K. Fukumoto, Y. Pennec, M. Bonfim, S. Pizzini, F. Petroff, A. Fontaine, J. Kirschner and J. Camarero, Journal of Applied Physics, 95, 6533 (2004).

$6 \quad$ X. W. Wu, J. Stohr, V. S. Pribiag, Y. Acremann, A. A. Tulapurkar, T. Tyliszczak, K. W. Chou, B. Bräuer, Z. P. Li, O. J. Lee, P. G. Gowtham, D. C. Ralph and R. A. Buhrman, Physical Review Letters, 106, 167202 (2011).

7 A. Fernández-Pacheco, D. Petit, R. Mansell, R. Lavrijsen, J. H. Lee and R. P. Cowburn, Physical Review B, 86, 104422 (2012).

8 S. A. Crooker, M. Furis, X. Lou, C. Adelmann, D. L. Smith, C. J. Palmstrom and P. A. Crowell, Science, 309, $2191(2005)$.

9 R. A. Rosenberg, S. Zohar, D. Keavney, R. Divan, A. Mascarenhas and M. A. Steiner, Review of Scientific Instruments, 83, 073701 (2012).

10 M. C. Hickey, C. D. Damsgaard, I. Farrer, S. N. Holmes, J. B. Husmann, C. S. Jacobsen, D. A. Ritchie, G. A. Lee, C. Jones and M. Pepper, Applied Physics Letters, 86, 252106 (2005).

11 K. Theis-Brohl, R. Scheidt, T. Zeidler, F. Schreiber and H. Zabel, Physical Review B, 53, 11613 (1996).

12 Y. B. Xu, T. M. Kernohan, D. J. Freeland, A. Ercole, M. Tselepi and J. A. Bland, Physical Review B, 58, 890 (1998 ).

13 N. Metoki, W. Donner and H. Zabel, Physical Review B, 49, 17351 (1994 ).

14 J. Daval and D. Randet, IEEE Transaction on Magnetics, 6, 768 (1970). 

Physical Review Letters, 75, 152 (1995).

16 A. Hubert and R. Schafer, Magnetic Domains: The Analysis of Magnetic Microstructures, Berlin: Springer, 1998.

17 R. C. O'Handley, Modern magnetic materials: Principles and Applications, New York: Wiley, 2000. $\mathrm{nm}<\mathrm{tCr}<2.3 \mathrm{~nm}$ and for videos of converging Co domain walls in $\mathrm{Cr}$ thickness range $1.1<\mathrm{tCr}<1.7 \mathrm{~nm}$ that collapse the domain at the Co switching field maxima in Figure 3(b).

19 B. Dieny, J. P. Gavigan and J. P. Rebouillat, Journal of Physics: Condensed Matter, 2, 159 (1990). 


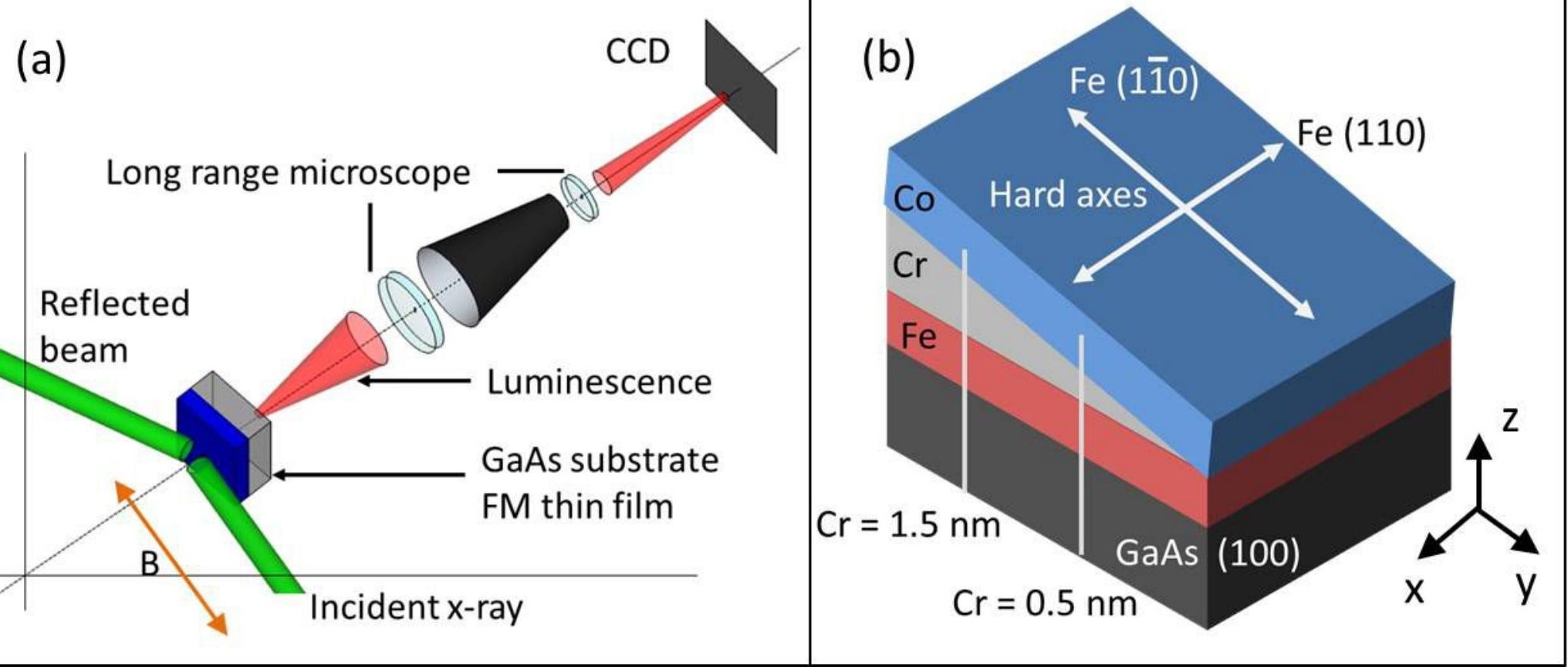




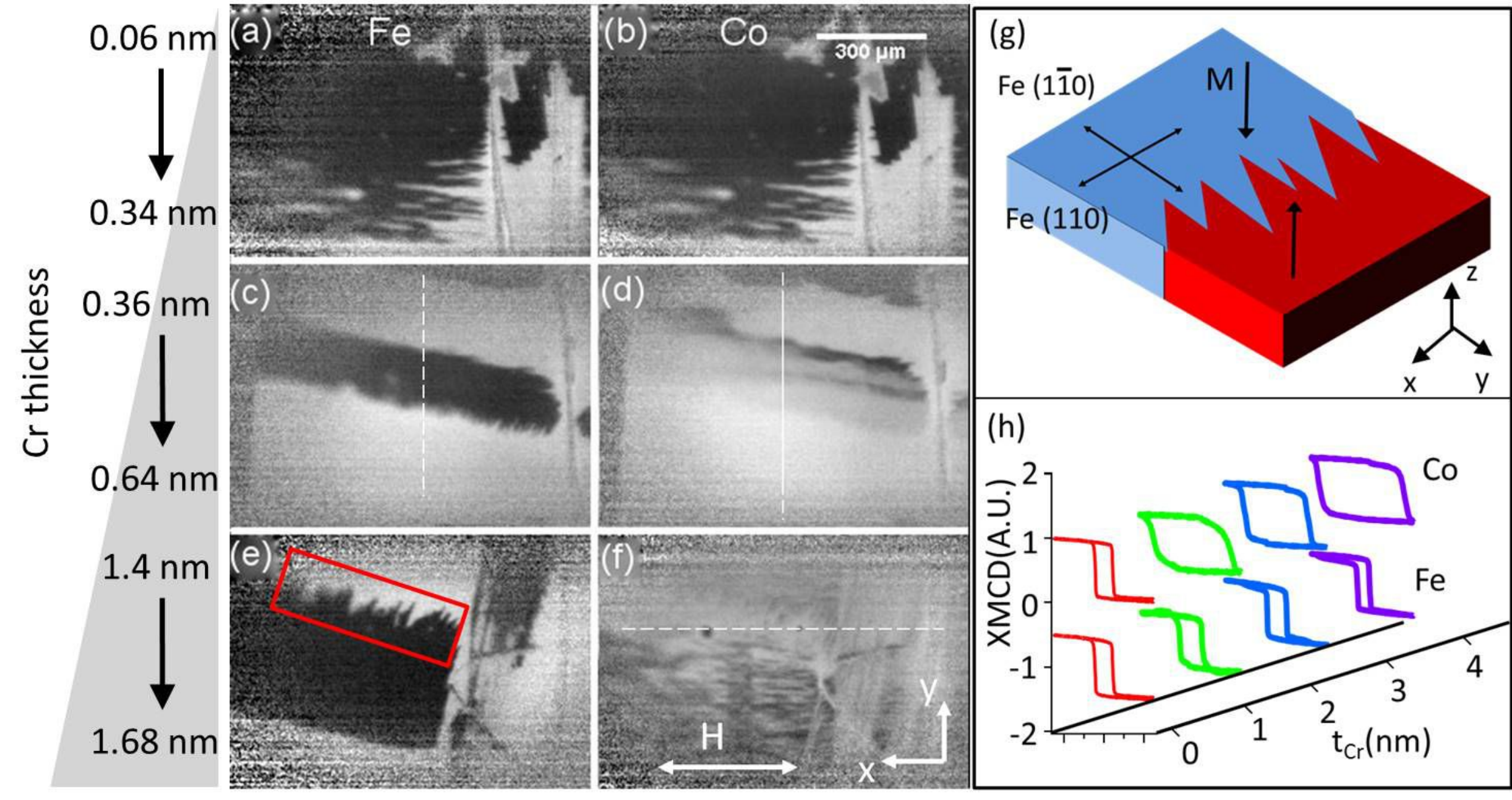


